\title{
Onset of convection due to horizontal laser beams
}

\author{
R. D. Boyd and C. M. Vest
}

Department of Mechanical Engineering, The University of Michigan, Ann Arbor, Michigan 48104

(Received 9 December 1974)

\begin{abstract}
When a horizontal $\mathrm{cw}$ laser beam is suddenly turned on in a quiescent isothermal fluid there is a delay time $t^{*}$ during which thermal blooming is governed by absorption of radiation and thermal conduction. For $t>t^{*}$, free convection becomes a significant factor. $t^{*}$ is determined by an approximate quasisteady hydrodynamic stability analysis and is compared with existing experimental data.

PACS numbers: $42.60 . \mathrm{N}, 45.25$.
\end{abstract}

Thermal blooming, the self-induced distortion of laser beams caused by heating of fluids due to absorption of energy from the beam, has been the subject of several experimental and analytical investigations. Most existing analyses of transient thermal blooming of $\mathrm{cw}$ laser beams are valid only for a small, but undefined, time interval after the beam is turned on. The objective of this letter is to predict the length of this time interval, which we refer to as the delay time and denote by $t^{*}$.

Suppose that a horizontal cw laser beam suddenly begins to propagate through a quiescent isothermal fluid. Initially, absorption of radiation and thermal conduction will be the only significant transport mechanisms, and the temperature and irradiance fields in the fluid will be radially symmetric about the axis of the beam. We refer to this as the conduction regime. Thermally induced density gradients cause the fluid to be unstable in the presence of a gravitational field; hence, after a time $t^{*}$, significant free convection will be initiated and asymmetric temperature and irradiance fields will result. In the work which follows, $t^{*}$ is estimated by an approximate quasisteady hydrodynamic stability analysis of the conduction regime.

The irradiance distribution in the cross section of the undisturbed laser beam is assumed to be Gaussian and, neglecting the effect of a finite aperture, can be expressed as

$$
I(r)=I_{0} \exp \left(-r^{2} / a^{2}\right),
$$

where $a$ is the characteristic radius of the beam. If $\alpha$ is the thermal absorptivity of the fluid at the laser wavelength, the thermal energy equation which governs the temperature field during the conduction regime is

$$
\frac{1}{\kappa} \frac{\partial \theta}{\partial t}=\frac{\partial}{\partial r}\left(r \frac{\partial \theta}{\partial r}\right)+\frac{a}{k} I_{0} \exp \left(-r^{2} / a^{2}\right),
$$

where $\kappa$ is the thermal diffusivity, $\theta=T(r)-T_{\infty}$ is the temperature excess above ambient, and $k$ is the thermal conductivity. We have assumed that $\alpha \ll 1$, so that axial variations of $I$ and $\theta$ can be neglected. The solution of Eq. (1), subject to the conditions

$$
\theta(r, 0)=0, \quad \theta(\infty, t)=0, \quad \text { and } \quad \lim _{r \rightarrow 0} r \frac{\partial \theta}{\partial r}=0,
$$

which can be found by Fourier transform or similarity analysis, is

$$
\theta(\rho, \tau)=-\frac{a^{2} \alpha I_{0}}{4 k}\left[E_{t}\left(\frac{-\rho^{2}}{4 \tau+1}\right)-E_{i}\left(-\rho^{2}\right)\right]
$$

$E_{i}$ is the exponential integral function, $\rho \equiv r / a$ is a dimensionless radius, and $\tau \equiv \kappa t / a^{2}$ is dimensionless time. This solution was previously given by Gordon et $a l{ }^{1}$

Several investigator $\mathrm{s}^{2-5}$ have observed that after a period of time the cross-sectional irradiance distribution becomes asymmetric, which indicates that freeconvection effects have become significant, so that Eq. (3) is no longer an adequate description of the temperature field. The initial asymmetry is most pronounced near the top of the beam. These observations, as well as studies of very similar temperature fields near suddenly heated horizontal wires, ${ }^{6}$ suggest that significant convection is initiated in the fluid in the upper-half of the beam. Although the fluid is always globally unstable, we hypothesize that significant convection does not occur until some critical density dis tribution, in which hydrodynamic disturbances are rapidly amplified, develops directly above the centerline of the beam. According to this hypothesis, a local quasisteady stability analysis of the fluid in this region is appropriate for estimating $t^{*}$.

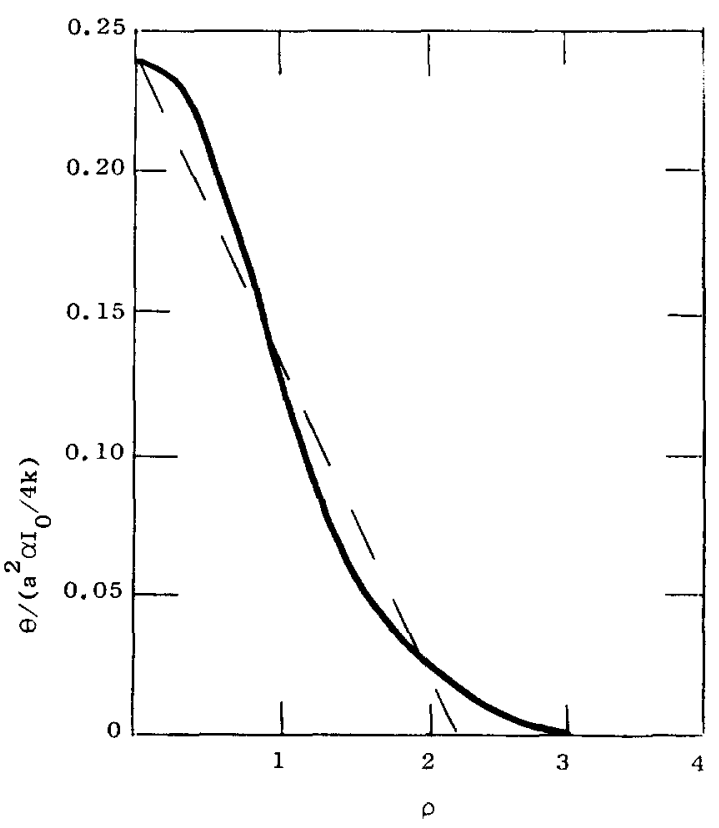

FIG. 1. Linear approximation of the temperature field at a typical time, $\tau=0.40$. The solid curve is the temperature field given by Eq. (3) and the dashed line is the linear approximation. 


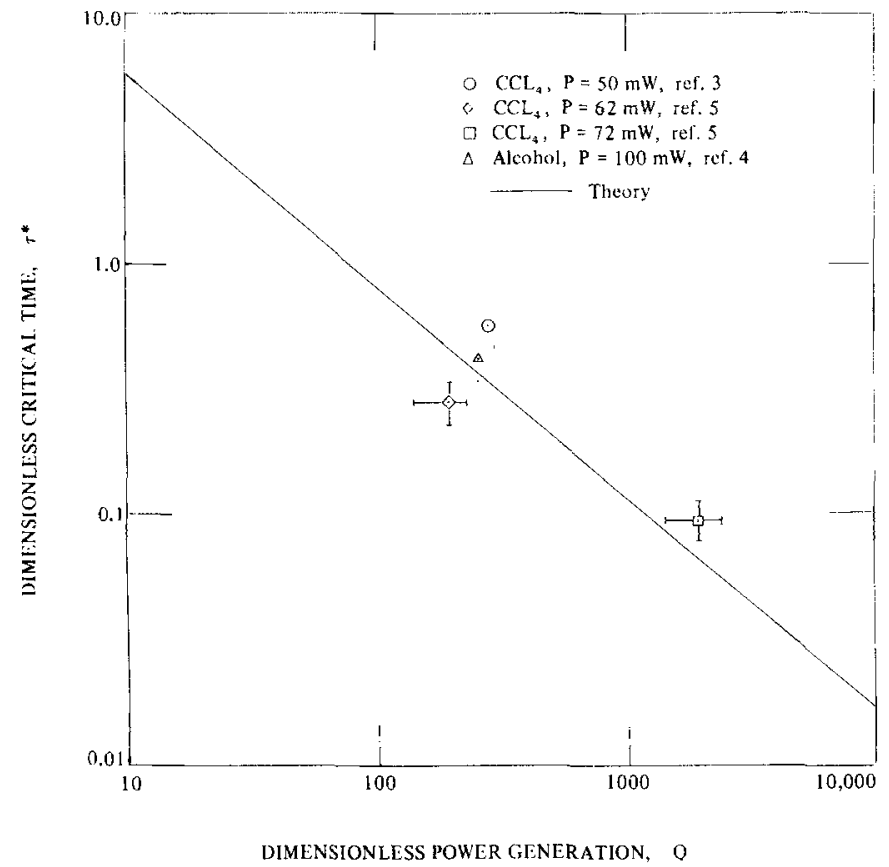

FIG. 2. Dimensionless critical time below which thermal blooming is governed by conduction and above which free convection is a significant factor. The solid line is predicted by an approximate hydrodynamic stability analysis. $\mathrm{O} ; \mathrm{CCl}_{4}$, $P=50 \mathrm{~mW}$, Ref, 3. $\diamond ; \mathrm{CCl}_{4}, P=62 \mathrm{~mW}$, Ref. 5. $\square ; \mathrm{CCl}_{4}$, $P=72 \mathrm{~mW}$, Ref. 5. $\Delta$; alcohol, $P=100 \mathrm{~mW}$, Ref. $4 .-$ theory.

The temperature is a maximum at the centerline and decreases upward, so that locally the density configuration is similar to that of a layer of fluid heated from below. The analysis of the stability of an infinite fluid layer of depth $\delta$ heated from below is classical and is known as the Benard problem. ${ }^{7}$ If the vertical temperature gradient is constant, and if shear is neglected at the upper and lower boundaries, a horizontal layer of fluid becomes linearly unstable at a critical Rayleigh number given by

$$
R a_{c}=g \gamma \beta \delta^{4} / \kappa \nu=658 .
$$

$\beta$ is the magnitude of the temperature gradient and $\nu$ is the kinematic viscosity of the fluid. Convection at Rayleigh numbers which are not greatly in excess of this value causes a multicellular motion with adjacent cells rotating in opposite senses. Similar cellular motions have been observed during the development of convection above suddenly heated horizontal wires. ${ }^{8}$

We hypothesize that convective effects become significant in thermal blooming only after the fluid directly above the center of the beam becomes unstable accord- ing to a criterion given approximately by Eq. (4), with an appropriate definition of $\beta$. The instantaneous temperature gradient, $\beta(\tau)$, was chosen to be that of an equivalent linear distribution having the same centerline temperature and integral as that of the actual profile. Figure 1 is a plot of the actual temperature profile and its linear approximation at $\tau=0.40$. The instantaneous temperature gradient $\beta$ and equivalent layer thickness (or penetration depth) $\delta$ are given by

$$
\begin{aligned}
& \beta(\tau)=-\theta^{2}(0, \tau)\left[2 a \int_{0}^{\infty} \theta(\rho, \tau) d \rho\right]^{-1} \\
& \delta(\tau)=\left[2 a \int_{0}^{\infty} \theta(\rho, \tau) d \rho\right][\theta(0, \tau)]^{-1},
\end{aligned}
$$

where $\theta(\rho, \tau)$ is given by Eq. (3)。The delay time $\tau^{*}$ is that for which

$$
R a=\left(\pi k / a^{3} \alpha P\right) Q \beta \delta^{4}=658,
$$

where $Q=g \gamma a^{3} \alpha P / \pi k \kappa \nu$ and $P=\pi a^{2} I_{0}$ represents the laser power. By computing $R a(\tau)$ for fixed $Q$, values of $\tau^{*}$ were determined according to $\mathrm{Eq}$. (7). The results of these computations are shown as a solid curve in Fig. 2.

We were able to glean sufficient data from reported experimental studies to provide four data points for comparison with the predicted values of $\tau^{*}$. These are indicated in Fig. 2. The experimental estimates of $\tau^{*}$ were based on the time at which the diameter of the top of the beam began to decrease. Although these data are limited, they appear to be in reasonable agreement with the predicted values of $\tau^{*}$. This agreement suggests that the concept of an appropriately defined critical Rayleigh number below which convective effects can be neglected in thermal blooming is valid, and that the delay times indicated in Fig. 2 are reasonable estimates of those which will occur in practice.

${ }^{1}$ J.P. Gordon, R.C.C. Leite, R.S. Moore, S.P.S. Porto, and J.R. Whinnery, J. Appl. Phys. 36, 3 (1965).

${ }^{2}$ J.R. Whinnery, D.T. Miller, and F. Dabby, EEE J. Quantum Electron. QE-3, 382 (1967).

${ }^{3}$ E. A. McLean, L. Sica, and A.J. Glass, Appl. Phys. Lett. 13, 369 (1968).

${ }^{4}$ S. A. Akhmanov, D. P. Krindach, A.V. Migulin, A. P. Sukhorukov, and R.V. Khokhlov, IEEE J. Quantum Electron. $\mathrm{QE}-4,568$ (1968).

${ }^{5}$ R. L. Carman and P. L. Kelley, Appl. Phys. Lett. 12, 241 (1968).

${ }^{6}$ C.M. Vest and M. L. Lawson, Int. J. Heat Mass Transfer 15, 1281 (1972).

${ }^{7} \mathrm{~S}$. Chandrasekhar, Hydrodynamic and Hydromagnetic Stability (Oxford U. P., London, 1961), pp. 35-36.

${ }^{8}$ W. Hauf and U. Grigull, in Advances in Heat Transfer, edited by J.P. Hartnett and T.F. Irvine, Jr. (Academic, New York, 1970), Vol. 6, pp. 134-366. 